\title{
Angular Correlation and Polarization Studies for Radiative Electron Capture into High-Z Ions
}

\author{
Th. Stöhlker*, D. Banas $\dagger$, S. Fritzsche**, A. Gumberidze*, C. Kozhuharov, \\ X. Ma ${ }^{\S}$, A. Orsic-Muthig*, U. Spillmann*, D. Sierpowski" ${ }^{\Uparrow}$, \\ A. Surzhykov**, S. Tachenov* and A. Warczak ${ }^{\mathbb{I}}$ \\ ${ }^{*}$ GSI-Darmstadt, 64291 Darmstadt and IKF, University of Frankfurt, Germany. \\ ${ }^{\dagger}$ Institute of Physics, Swietokrzyska Academy, 25-406 Kielce, Poland. \\ **Fachbereich Physik, Universität Kassel, Heinrich-Plett-Str. 40, D - 34132 Kassel, Germany. \\ ${ }^{\ddagger}$ Gesellschaft für Schwerionenforschung (GSI), D-64291 Darmstadt, Germany. \\ ${ }^{\S}$ Institut for Modern Physics, Lanzhou, China. \\ IJagiellonian University, Cracow, Poland
}

\begin{abstract}
Recent photon correlation studies for Radiative Electron Capture into high-Z projectiles are reviewed. Emphasis is given to the investigation of polarization phenomena which are now accessible due to recent developments in position sensitive solid-states detectors. It is shown, that REC may provide a tool for the diagnostics and detection of the spin-polarization of particles involved in atomic collisions. Also the impact of REC studies for atomic structure studies is outlined. Here the strong alignment of excited states induced by REC allowed us to observe an interference between competing decay branches for the case of the Lyman- $\alpha_{1}$ transition in hydrogen-like ions.
\end{abstract}

\section{INTRODUCTION}

In atomic collisions of highly-charged heavy ions and low-Z target atoms the coupling between the electron and the electromagnetic field of the fast moving projectile results in an electron capture into a bound state of the ion via simultaneous emission of a photon $[1,2,3]$. Consequently this process (Radiative Electron Capture, REC) can be viewed as the time-reversed photoionization process (see Fig. 1) $[2,3,4,5,6]$. Up to now, the most detailed studies were performed for the heaviest ions such as bare uranium $[7,8,9]$. Here angular-distribution experiments performed at the storage ring ESR showed that REC is a powerful tool for precision studies of the photoionization process in the high$Z$ domain. This technique allowed us to extend the information about photoionization to much lower energies than those accessible for neutral heavy elements in the direct reaction channel, demonstrating that higher-order multipole contributions and magnetic corrections persist even at energies close to the threshold. The data obtained also prove that REC leads to strongly aligned projectile states. This enabled the observation of an interference between the leading $\mathbf{E} 1$ decay channel and the weak $\mathbf{M} 2$ branch for the case of the Lyman- $\alpha_{1}\left(2 p_{3 / 2} \rightarrow 1 s_{1 / 2}\right)$ transition in hydrogen-like uranium [10,11].

A topic which has recently attracted particular attention is the linear polarization of the REC photons $[12,13]$. Theoretical investigations point out that REC is a source 

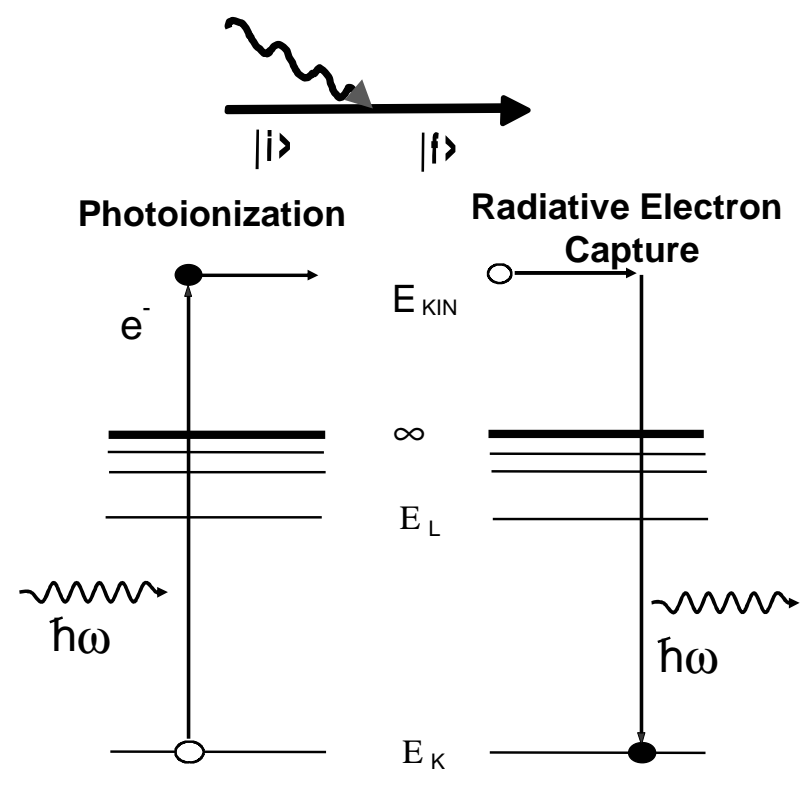

FIGURE 1. Radiative Electron Capture can be viewed as the time-reversed photoionization which results in an electron capture into a bound state of the ion via simultaneous emission of a photon.

of strongly linearly polarized high-energetic photons, with a high sensitivity on the details of the REC process. Most important and in contrast to angular-differential cross sections, it turned out that the polarization of the emitted photons appears to be strongly affected by a possible spin-polarization of the particles involved into the collision [14]. Similar effects has long been known for the atomic photoeffect $[15,16]$. In fact, despite the difficulty to detect the linear polarization of high-energetic photons, such measurements seem to be possible nowadays due to the recent developments in position-sensitive solid-state detectors [17].

In the following we concentrate on REC into excited states, and the identification of an interference between the $\mathbf{E} 1$ and $\mathbf{M} 2$ transition amplitudes in the decay of the $2 \mathrm{p}_{3 / 2}$ level in hydrogen-like heavy ions. This is followed by a discussion of a first linear polarization measurement for REC into the K-shell of bare uranium. Also the the influence of spin polarized electrons on the linear polarization of the REC photons is discussed.

\section{REC INTO EXCITED STATES: ALIGNMENT STUDIES}

In the past, the angular distribution of this the Lyman- $\alpha_{1}$ transition $\left(2 \mathrm{p}_{3 / 2} \rightarrow 1 \mathrm{~s}_{1 / 2}\right)$ in high- $Z$ ions was subject of intense experimental and theoretical studies in order to elucidate the magnetic substate population of REC in relativistic collisions of bare uranium with low-Z target atoms [4, 10] (compare Fig. 2).

If the magnetic sublevels with different absolute magnetic quantum numbers $\mu$ are populated non-statistically in collisions of ions with electrons or atoms, alignment occurs leading to an anisotrope emission pattern. For the particular case of the $2 p_{3 / 2}$ state this distribution is given by [4]:

$$
W(\theta) \propto 1+\beta_{20}\left(1-\frac{3}{2} \sin ^{2} \theta\right)
$$




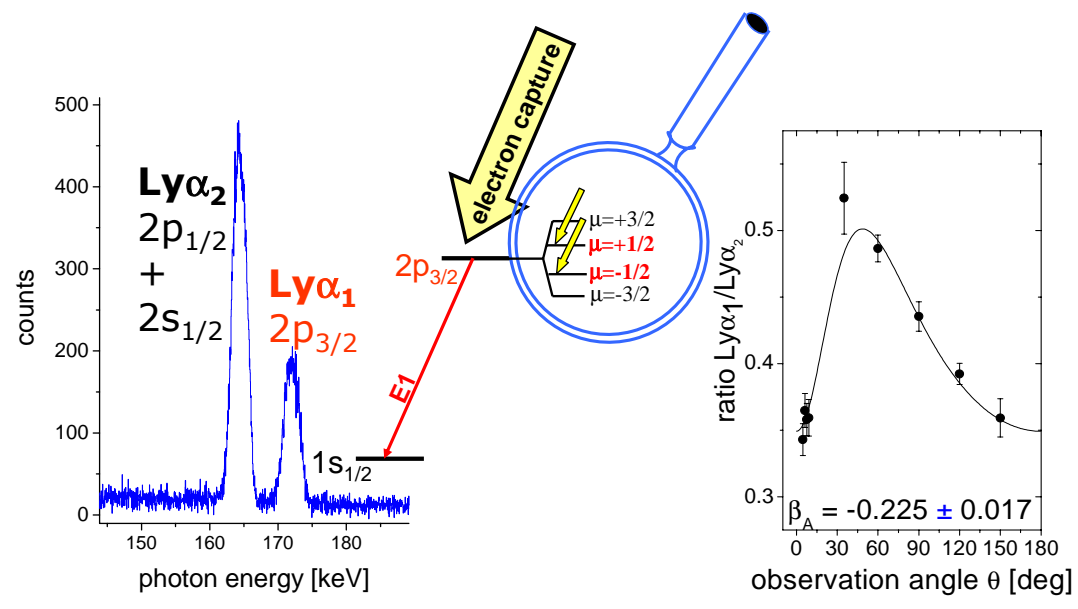

FIGURE 2. Left side: Lyman- $\alpha_{2}\left(2 \mathrm{p}_{1 / 2}, 2 \mathrm{~s}_{1 / 2} \rightarrow 1 \mathrm{~s}_{1 / 2}\right)$ and Lyman $-\alpha_{1}$ transition $\left(2 \mathrm{p}_{3 / 2} \rightarrow 1 \mathrm{~s}_{1 / 2}\right)$ of $\mathrm{U}^{91+}$ as observed at an observation angle $\theta_{l a b}$ of $132^{\circ}$ for $\mathrm{U}^{92+} \rightarrow \mathrm{N}_{2}$ collisions at $300 \mathrm{MeV} / \mathrm{u}$. Contrary to the Lyman- $\alpha_{2}$ line, the Lyman $-\alpha_{1}$ transition exhibits a strong intensity variation as function of the observation angle caused by a preferred population of the magnetic sublevels $\mu \pm \frac{1}{2}$ (see right side) [10].

where $\theta$ is the angle between the direction of the excitation photon and the beam direction (emitter frame) while $\beta_{20}$ denotes the anisotropy coefficient. In the case of the $2 \mathrm{p}_{3 / 2}$ level, the anisotropy coefficient can be expressed as:

$$
\beta_{20}=\frac{1}{2} \frac{\sigma\left(\frac{3}{2}, \pm \frac{3}{2}\right)-\sigma\left(\frac{3}{2}, \pm \frac{1}{2}\right)}{\sigma\left(\frac{3}{2}, \pm \frac{3}{2}\right)+\sigma\left(\frac{3}{2}, \pm \frac{1}{2}\right)},
$$

Here $\sigma\left(3 / 2, \mu_{n}\right)$ describes the population of substate $\mu_{n}$ of the $2 \mathrm{p}_{3 / 2}$ level. Based on expression (2), the theoretical and observed angular distributions were compared in detail and a remarkable variance was found $[4,10]$. Viewing the used rigorous relativistic calculations [2] in more detail one finds that it is assumed that the level of interest namely the $2 \mathrm{p}_{3 / 2}$ state decays solely by an electric dipole transitions. At high-Z, however, the magnetic quadrupole decay $\mathbf{M} 2$ may contribute in addition and may affect considerably the emission (angular distribution and polarization) of the decay photons.

By using the density matrix theory one can consider the magnetic (M2) decay branch in a coherent way. As a result, the alignment coefficient parameter $\beta_{20}$ must now be replaced by the product $\beta_{20} \cdot f(E 1, M 2)$ where $f(E 1, M 2)$ is a structure function which takes into account the interference effect. This structure function is given by

$$
f(E 1, M 2) \propto\left[1+2 \sqrt{3} \frac{<\|M 2\|>}{<\|E 1\|>}\right]
$$

where $\langle\| E 1||\rangle$ and $\langle\|M 2\|\rangle$ are the reduced matrix elements for the electric and the magnetic bound-bound multipole transitions, respectively [18, 19].

Considering the dipole term only, i.e. $\langle\| M 2||\rangle \approx 0$, we obtain $f(E 1, M 2) \equiv 1$. As seen from Eq. 3, the main correction to this dipole approximation arises from the term 

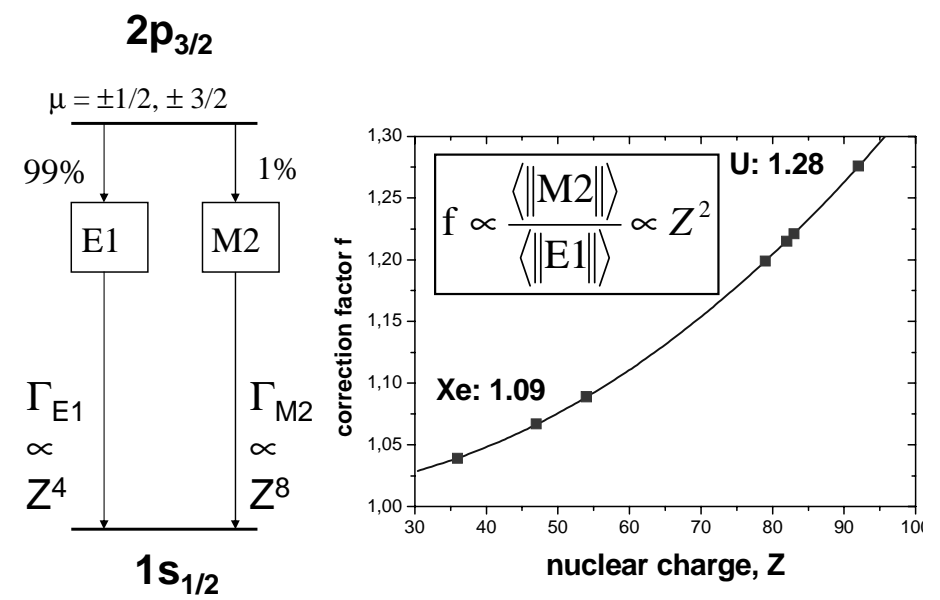

FIGURE 3. Structure part $f(E 1, M 2)$ (Eq. 3 ) as calculated for the decay of the $2 \mathrm{p}_{3 / 2}$ state in hydrogenlike ions as function of the nuclear charge $Z$ [11]. The dependence as function of the nuclear charge $Z$ is roughly reproduced by a $Z^{2}$ scaling.

which is proportional to the ratio of the transition amplitudes $\langle\| M 2||\rangle /\langle\| E 1||\rangle$. For high $-\mathrm{Z}$ ions this ratio is of the order $\sim 0.1$, leading to a $1 \%$ contribution of the $\mathbf{M} 2$ component to the total decay rate. Indeed, even for hydrogen-like uranium $(Z=92)$ the E1 transition rate amounts to $\Gamma_{\mathrm{E} 1}=3.92 \cdot 10^{16} s^{-1}$ whereas the $\mathbf{M} 2$ rate $\Gamma_{\mathrm{M} 2}=$ $2.82 \cdot 10^{14} s^{-1}$ contributes less than $1 \%$ to the total decay rate. Up to now this rather small contribution was the main justification why the M2 component of the radiation field has not been incorporated in computations on the $2 \mathrm{p}_{3 / 2}$ decay of hydrogen-like ions or similar groundstate transitions in the high $-Z$ regime $[23,24]$. Figure 3 shows the structure function (3) for hydrogen-like ions as a function of the charge Z. For $\mathrm{H}$-like uranium, this dimensionless function is as large as 1.28. Since this function basically depends on the ratio $\langle\| M 2||\rangle /\langle\| E 1||\rangle$ of the reduced matrix elements, its scaling follows roughly a $\mathrm{Z}^{2}$ dependence. Obviously, this deviates markedly from a $\mathrm{Z}^{4}$ scaling as one might expect it from the corresponding decay rates. As a consequence the interference leads to an non-negligible effect of a few percent even for medium-Z ions (compare Fig. 3).

In Fig. 4 we compare the experimental results for H-like uranium (solid points) with the corresponding theoretical findings (full line) and the results obtained assuming $f(E 1, M 2)=1$ (dashed line), i.e. neglecting the interference term. From the figure it is evident that the former departure of the theoretical results from the experimental values is removed by taking the interference term into account. This proves the importance of the interference between the $\mathbf{E} 1$ and $\mathbf{M} 2$ decay branches for the decay of $2 \mathrm{p}_{3 / 2}$ state.

\section{PHOTON POLARIZATION STUDIES}

The theoretical studies performed very recently revealed the sensitivity of the linear photon polarization to the presence of spin-flip effects which lead to a depolarization of the 


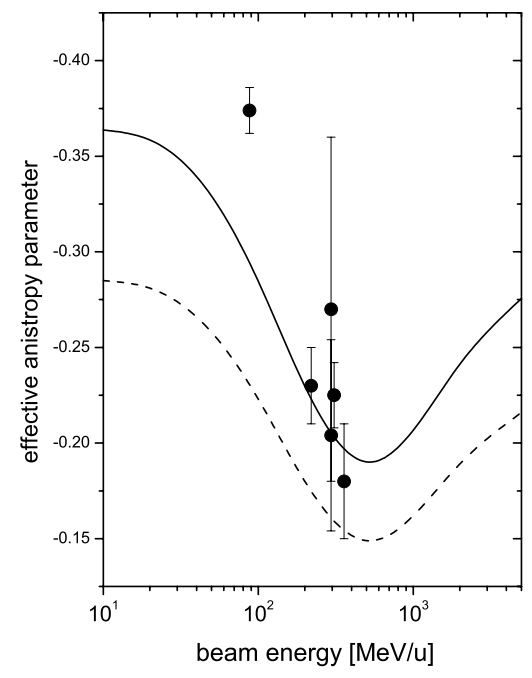

FIGURE 4. The experimentally determined effective anisotropy parameters $\beta_{20}^{\text {eff }}$ (solid points) for the Lyman- $\alpha_{1}$ radiation of $U^{91+}$ produced in $U^{92+} \rightarrow N_{2}$ collisions as a function of collision energy [10]. The dashed line represents the theoretical predictions for $f(E 1, M 2) \equiv 1$. The solid line shows the corresponding result which considers the interference term $f(E 1, M 2)$, i.e. $\beta_{20} \cdot f(E 1, M 2)[11]$.

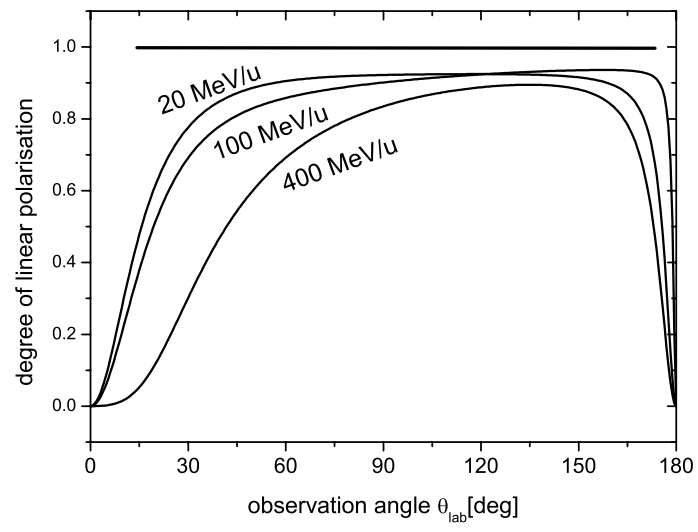

FIGURE 5. Degree of linear polarization of the emitted photon as a function of the laboratory photon angle for the $K$-shell RR of $U^{92+}$ projectiles in collisions with unpolarized electrons [12, 13]. The straight line refers to the non-relativistic dipole approximation which predicts a $100 \%$ polarization regardless of the observation angle.

$\mathrm{X}$-ray emission in particular in the forward hemisphere [12, 13, 14]. This is depicted in Fig. 5 where the effect of depolarization is clearly visible when comparing the degree of linear-polarization predicted for $\mathrm{U}^{92+} \rightarrow \mathrm{e}^{-}$at various collision energies. Experimentally, however, linear polarization data for REC or radiative recombination are not available at all. At high-Z, i.e. photon energies above $100 \mathrm{keV}$, this topic can be addressed experimentally with high efficiency by a new generation of segmented germanium detectors 


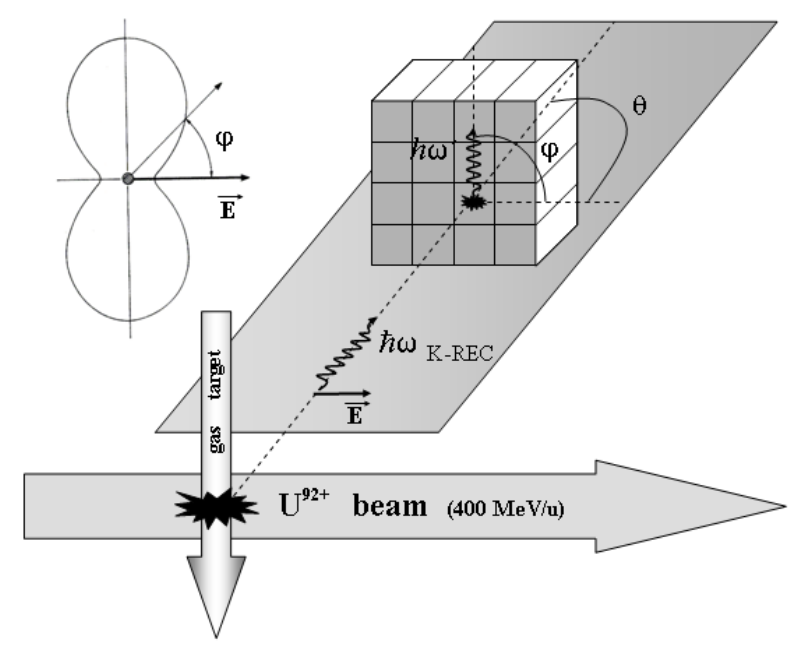

FIGURE 6. Detector geometry used for the measurement of the linear photon polarization for K-REC at $400 \mathrm{MeV} / \mathrm{u} \mathrm{U}^{92+} \rightarrow N_{2}$ collisions by exploiting the Compton effect.

allowing for energy as well as position resolution. Here polarization measurements can be performed by exploiting the dependence of the differential Compton scattering crosssection on the linear polarization of the initial photon (see e.g. [21, 22]). Following the Klein-Nishina formula, the differential cross-section for Compton scattering of a photon with initial energy $\hbar \omega$ is given by

$$
\frac{d \sigma}{d \Omega}=\frac{1}{2} r_{0}^{2}\left(\frac{\hbar \omega^{\prime}}{\hbar \omega}\right)^{2}\left(\frac{\hbar \omega^{\prime}}{\hbar \omega}+\frac{\hbar \omega}{\hbar \omega^{\prime}}-2 \sin ^{2} \theta \cos ^{2} \varphi\right) .
$$

where $\hbar \omega^{\prime}$ denotes the energy of the scattered photon, $\theta$ the angle between the initial and the scattered photon, and $\varphi$ the angle between the polarization vector of the initial photon and the propagation direction of the scattered one (compare Fig. 6).

For bare uranium ions (400 MeV/u) a polarization study for REC into the K-shell has recently been performed at the jet-target of the storage ring ESR. For this purpose a $4 \times 4$ planar germanium pixel detector with a pixel size of $7 \times 7 \mathrm{~mm}$ has been used [20], mounted at an observation angle of $90^{\circ}$ (for the geometry of the experiment compare Fig. 6). In the experiment the photon polarization can be derived from a coincident registration of events occurring simultaneously in two pixels. One pixel registers the Compton recoil electron $(\Delta E)$ and the other the outgoing scattered photon $\left(\hbar \omega^{\prime}\right)$. In Fig. 7a a scatter plot of such coincident photon events is displayed. The intense diagonal reflects a constant sum energy equal to the K-REC transition, i.e. $\mathrm{E}_{K-R E C}=\Delta E+\hbar \omega^{\prime}$. It is important to mention that for our initial energies $\left(\mathrm{E}_{K-R E C} \approx 250 \mathrm{keV}\right)$ the condition $\Delta E<\hbar \omega^{\prime}$ is always fulfilled which allows us to identify the segment where scattering took place. The latter also explains the two maxima present in the $2 \mathrm{D}$ scatter plot. In Fig. $7 b$ we compare the coincident sum energy spectrum for scattering parallel $\left(\mathrm{I}_{\|}\right)$and perpendicular $\left(\mathrm{I}_{\perp}\right)$ to the scattering plane (defined by the ion beam and K-REC photon). From this figure we can already state that the K-REC radiation is strongly polarized 

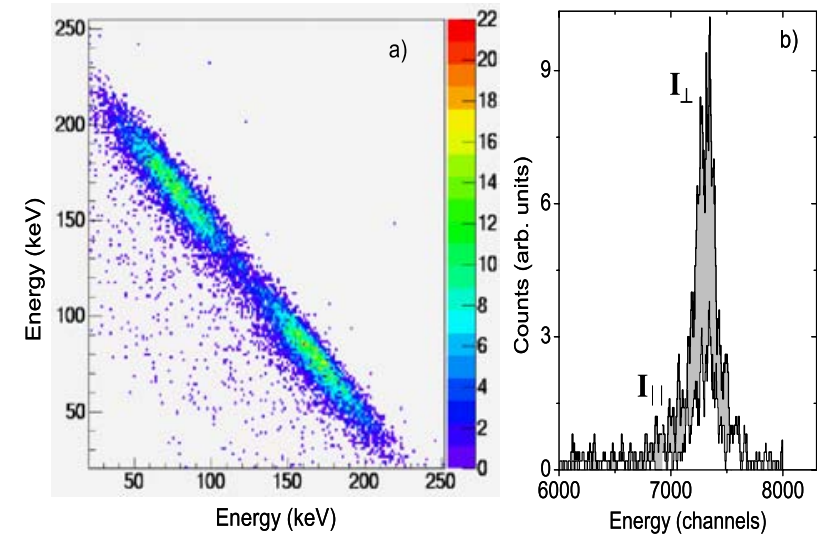

FIGURE 7. a) Scatter plot of coincident Compton events; b) the coincident sum energy spectrum for scattering parallel $\left(\mathrm{I}_{\|}\right.$, white area) and perpendicular $\left(\mathrm{I}_{\perp}\right.$, shaded area) to the scattering plane.

within the scattering plane. An accurate value for the linear degree of polarization can finally be obtained by analyzing all the different combinations of pixel pairs. In addition either Monte Carlo simulations and/or calibration measurements using $\gamma$-transitions with known polarization are required to determine the polarization sensitivity of the used detector system with high accuracy.

\section{THE EFFECT OF SPIN-POLARIZED PARTICLES}

The polarization collision experiments require an effective tool for the diagnostics of the polarization properties of the particles involved into the collision (projectile, electrons or target atoms). As pointed out very recently, REC can be attributed as a "probe" process, whose linear polarization is sensitive to the polarization states of the collision system [14]. From an experimental viewpoint, the polarization of the emitted photons are most easily described in terms of the Stokes parameters. While the parameter $P_{1}=$ $\left(I_{0^{o}}-I_{90^{\circ}}\right) /\left(I_{0^{o}}+I_{90^{\circ}}\right)$ is obtained from the intensities parallel and perpendicular to the reaction plane (as defined by the directions of the incoming ion beam and the emitted photons), the parameter $P_{2}$ follows from a similar intensity ratio, taken at $45^{\circ}$ and $135^{\circ}$, respectively. Thus, the two parameters $P_{1}$ and $P_{2}$ together characterize completely the linear polarization with respect to the collision plane, i.e. its degree and orientation. Figure 8 displays the Stokes parameters $P_{1}$ and $P_{2}$ of the recombination/REC photons, following capture into the $\mathrm{K}$-shell of bare uranium ions. For capture of unpolarized electrons, only the Stokes parameter $P_{1}$ is non-zero and positive, while $P_{2}$ is identically zero. This implies that, for unpolarized electrons, the polarization of the REC photons is always within the reaction plane. For the capture of polarized electrons the Stokes parameter $P_{2}$ becomes non-zero, in particular for small forward observation angles, while the parameter $P_{1}$ does not change. Apparently, this leads to an overall rotation of the linear polarization of the recombination/REC photons out of the reaction plane and may therefore serve as a valuable tool for "measuring" the polarization properties of 


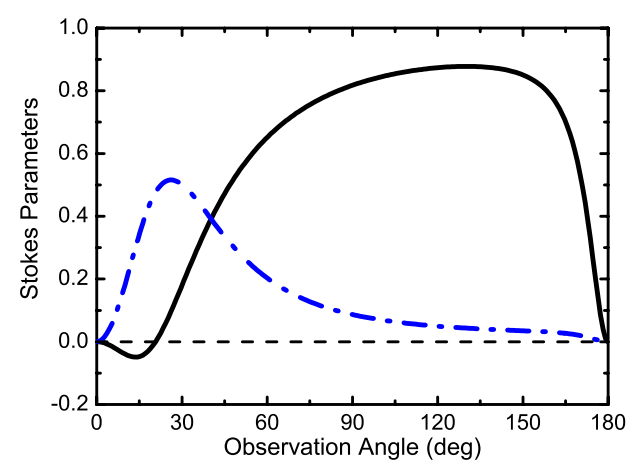

FIGURE 8. The Stokes parameters $P_{1}$ (solid line) and $P_{2}$ (dashed line) of recombination/REC photons for capture into the $K$-shell of the bare uranium ions at an energy of $500 \mathrm{MeV} / \mathrm{u}$ [14]. The Stokes parameters $P_{2}$ are shown for the capture of completely polarized electrons.

either the electrons or, respectively, of the ion beam if unpolarized electrons are captured by polarized ions.

\section{SUMMARY}

In summary, the fundamental interaction process between an electron and a photon in the presence of a strong central atomic field is discussed from the point of view of REC into highly-charged ions. For the case of capture into excited states it has been shown that by means of REC an interference between the leading $\mathbf{E} 1$ decay channel and the weak M2 branch was identified for the case of the Lyman- $\alpha_{1}$ decay in H-like uranium. We have to add the importance of this finding for any atomic or ionic system where beside the leading multipolarity a higher multipole order is allowed. Here, both the polarization and the angular distribution of the photon emission might be affected considerably. Furthermore, we discussed the current efforts to gain experimental information on the linear polarization of REC photons. Due to the recent developments of position sensitive solid detectors with imaging and timing capability for the hard x-ray regime, this challenging topic can now be addressed experimentally. This represents an important step towards more accurate studies of bound-bound and free-bound transitions in the realm of high-Z ions where relativistic structure and collision effects are predicted to influence strongly the linear polarization of the emitted x-rays. In particular, such studies may provide a diagnostic tool for the detection of spin-polarized particles involved in atomic collisions.

\section{ACKNOWLEDGMENT}

For the close collaboration we like to thank F. Bosch, R.W. Dunford, E. Kanter, S. Hagmann, R. Mann, P.H. Mokler, Z. Stachura and the members of the ESR team. 
The support by D. Protic and Th. Krings at IKP, FZ-Jülich for providing us with position sensitive Ge(i) detector system is gratefully acknowledged. Also we like to acknowledged the helpful discussions and the longstanding close collaboration with Jörg Eichler. This research has been supported by a Marie Curie Fellowship of the European Community Programme IHP under contract number HPMT-CT-2000-00197 and by the EU grant EC-HPRI-CT-1999-00001.

\section{REFERENCES}

1. J. Eichler and W.E. Meyerhof Relativistic Atomic Collisions (Academic Press, San Diego, 1995).

2. A. Ichihara, T. Shirai, and J. Eichler, Phys. Rev. A 54, 4954 (1996).

3. P. H. Mokler and Th. Stöhlker, Adv. in Atomic, Molecular, and Optical Physics, Vol.37, 297 (1996).

4. J. Eichler, Nucl. Phys. A 572, 147 (1994).

5. J. Eichler et al., Phys. Rev. A51, 3027 (1995).

6. Th. Stöhlker et al., Phys. Rev. A51, 2098 (1995).

7. Th. Stöhlker et al., Phys. Rev. A 58, 2043 (1998).

8. Th. Stöhlker et al., Phys. Rev. Lett. 82, 3232 (1999); 841360 (2000).

9. Th. Stöhlker et al., Phys. Rev. Lett. 86, 983 (2001).

10. Th. Stöhlker et al., Phys. Rev. Lett. 79, 3270 (1997).

11. A. Surzhykov, S. Fritzsche, A. Gumberidze, and Th. Stöhlker. Phys. Rev. Lett. 88, 153001 (2002).

12. A. Surzhykov, S. Fritzsche, Th. Stöhlker, Phys. Lett. A 289, 213 (2001).

13. J. Eichler and A. Ichihara, Phys. Rev. A 65, 062716 (2002).

14. A. Surzhykov et al., Phys. Rev. A 68, 22710 (2003).

15. R. Pratt et al., Phys. Rev. 134, A916 (1964).

16. J. Scofield, Phys. Rev. A 40, 3054 (1989).

17. Th. Stöhlker et al., Nucl. Instr. Meth. B 205, 210 (2003).

18. J. Eichler, A. Ichihara and T. Shirai, Phys. Rev. A 58 (1998) 2128.

19. G. Drake, Theory of Transitions, and the Electroweak Interaction, in: G. W. Series (Ed.), The Spectrum of Atomic Hydrogen: Advantages, (Word Scientific, Singapore, 1988).

20. Provided by D. Protic et al., IKP FZ-Jülich (2002).

21. K. Siegbahn, Alpha-, Beta-, and Gamma-Ray Spectroscopy (North Holland Publishing Company, New York, 1965).

22. S.E. Inderhees et al., IEEE Trans. Nucl. Science 43, 1467 (1996).

23. M. H. Chen and J. H. Scofield, Phys. Rev. A 52, 2057 (1995).

24. M. Gail, N. Grün, and W. Scheid, J. Phys. B 31, 4645 (1998). 